\title{
FDI Inflows, Foreign Trade and Conversion of Government Functions
}

\section{Huiai Yuan ${ }^{a}$, Lihong Zhao ${ }^{b}$ and Xiaona Zhong ${ }^{c}$}

\author{
Shanxi Institute of International Trade and Commerce, Xi'an Shanxi, China. \\ a yha1990@163.com, b lihong@163.com, cxnzhong91@163.com
}

Keywords: FDI; Trade; Government Final Consumption; VEC model; Granger causality.

\begin{abstract}
This paper empirically analyze relation of FDI inflows, foreign trade and government final consumption, using Johansen cointegration test, VEC model and Granger causality model by using the Chinese data from 1980 to 2016. The results shows that there is an equilibrium relationship that is negative effects in the short term and positive effects the in the long term between direct investment inflows and government final consumption, that is, FDI will prompt the conversion of government functions to the market in the short term, but it will not be conducive to the marketization of government functions in the long term; There is a long-term complementary relationship, which is a short-term substitute between FDI and trade. In economic development, there is a conflict between short-term goals and long-term goals, between the government and the market. Therefore, this paper points out, in the transformation and upgrading of China's economy, institutional reforms are needed, and making the government playing a key role in the allocation of economic resources, and the market gradually playing a decisive role.
\end{abstract}

\section{1. 前言}

中国在计划经济向市场经济转型的道路中，技术创新带来了前所未有的经济发展。技术创新 在整个人类历史发展起着决定性的力量, 但是作为经济体系的内生性变量, 其内部已处于均 衡状态。制度创新在经济增长长期发展中, 作为经济系统的外生性变量, 其是发展的动力源 泉, 是技术创新的前提, 是政府的主要职能。因此, 在经济结构转型升级过程中, 制度创新 就势在必行。自改革开放以来, 我国的对外开放度逐步提升, FDI 流入量从 1991 的 43.66 亿 美元增至 2002 年 527.4 亿美元， 2016 年 1337 亿美元，其在 26 年间增长了 31 倍, 可以说自 2002 年以来中国 FDI 流入量一直居于世界前列; 我国进出口总额达 36855.6 亿美元, 相较于 2013（41589.9 亿美元）、2014（43015.3 亿美元）、2015（3953.3 亿美元）年有所下降, 但 自 1978 年以来总体增长较为迅速; 政府最终消费支出 2016 年年增长率达 $9.4 \%$, 占最终消费 支出近为 $30 \%$ 。本文过对此三个经济变量进行实证分析, 着重在解决两个问题, 一是直接投资 与对外贸易在短期、长期究竟是互补关系还是替代关系, 二是直接投资的流入与政府职能的 转化是否存在短期与长期效应关系, 并对于出现的这种结果予以分析, 进而对中国的体制二 元化进行探讨, 归根结底就是理清政府与市场的关系.

\section{2. 模型建立及数据说明}

\section{1. 模型构建}

$$
\mathrm{G}=\mathrm{A}_{0} \mathrm{~F}^{\alpha} \mathrm{T}^{\beta}
$$

其中 $G$ 为政府最终消费, $F$ 为直接投资流入, $T$ 为对外贸易, $A_{0}$ 为影响政府最终消费的其他 因素, $\alpha 、 \beta$ 分别为 $F$ 和 $\mathrm{T}$ 的参数。

对 (1) 导入时间下标 $t$, 并对等式两边取对数, 可得到:

简化上述公式:

$$
\ln \mathrm{G}_{\mathrm{t}}=\ln \mathrm{A}_{0}+\alpha \ln \mathrm{F}_{\mathrm{t}}+\beta \ln \mathrm{T}_{\mathrm{t}}
$$

$$
g c_{t}=a_{0}+\alpha f d i_{t}+\beta \operatorname{tr}_{t}
$$




\section{2. 数据来源及说明}

中国直接投资流入的数据来自 UNCTAD stat (United Nations Conference Trade and Development stat），政府最终消费、居民最终消费、最终消费 [政府最终消费与居民最终消 费构成最终消费（根据《中国统计年鉴》 “支出法国内生产总值结构” 分栏）]，贸易（货物 和服务) 进口、出口和 GDP 的数据均来自世界银行, 数据的时间序列长度为 1980-2016 年。 其中直接投资流入、GDP 的数据以当年价格计算; 政府最终消费、居民最终消费、最终消费， 贸易 (货物和服务) 进口、出口数据是以 2005 年不变价格计算的实际值, 以上变量计价单 位均为美元。

\section{3. 单位根检验及协整关系检验}

本文计量部分的实证分析使用 Eviews7.0。本研究采用 ADF (Augmented Dickey Fuller Test) 统计量法来检验变量时间序列数据的平稳性, 表 1 为变量水平序列与差分序列的 ADF 单位根 检验结果。从检验结果可以看出: fdit 和 trt 的水平序列是非平稳序列, gct 的水平序列是 具有趋势的平稳序列, 但所有序列的一阶差分序列都在 5\%的显著水平下平稳, 即, 满足所有 变量是 I（1）的过程，为避免 “伪回归” 问题，接下来需进行协整关系检验.

\section{表1. ADF单位根检验结果}

\begin{tabular}{cccc|cccc|c}
\hline $\begin{array}{c}\text { 水平 } \\
\text { 序列 }\end{array}$ & $\begin{array}{l}\text { 检验 } \\
\text { 形式 }\end{array}$ & ADF & Prob. & $\begin{array}{c}\text { 差分 } \\
\text { 序列 }\end{array}$ & $\begin{array}{c}\text { 检验 } \\
\text { 形式 }\end{array}$ & ADF & Prob. & 结论 \\
\hline fdit & $\mathrm{C}, \mathrm{T}, 1$ & -1.099 & 0.913 & $\triangle \mathrm{fdi}$ & $\mathrm{N}, \mathrm{N}, 0$ & -5.495 & 0.000 & $\mathrm{I}(1)$ \\
$\mathrm{gct}$ & $\mathrm{C}, \mathrm{T}, 7$ & -4.324 & 0.011 & $\triangle \mathrm{gc}$ & $\mathrm{C}, \mathrm{N}, 5$ & -3.952 & 0.006 & $\mathrm{I}(1)$ \\
$\operatorname{trt}$ & $\mathrm{C}, \mathrm{T}, 1$ & -2.579 & 0.292 & $\triangle \mathrm{tr}$ & $\mathrm{N}, \mathrm{N}, 0$ & -5.473 & 0.000 & $\mathrm{I}(1)$ \\
\hline
\end{tabular}

注:（1）检验形式 (C, T, q) 分别代表截距项、趋势项和滞后阶数, 存在截距项或趋势项分别 记为 $\mathrm{C}$ 和 $\mathrm{T}$, 否则为 $\mathrm{N}$;

（2） $\triangle$ 表示一阶差分; （3）Prob.：ADF 的伴随概率。

本文使用 Johansen 协整关系检验法进行计量分析, 即在建立无约束 VAR Vector Autoregression) 模型的基础上进行协整关系检验。如表 2 所示, 5 种检验标准均选择了 5 作 为 VAR 模型的最优滞后阶数, 即: 最优滞后阶数确定为 5 。因此, Johansen 协整检验的最优 滞后阶数为 4 , 滞后区间为 $1-4$ 。

表2.VAR模型最优滞后阶数选择

\begin{tabular}{ccccccc}
\hline Lag & LogL & LR & FPE & AIC & SC & HQ \\
\hline 0 & 23.58677 & NA & $4.61 \mathrm{e}-05$ & -1.470484 & -1.327748 & -1.426848 \\
1 & 88.84710 & 111.8748 & $8.34 \mathrm{e}-07$ & -5.489079 & -4.918134 & -5.314535 \\
2 & 123.3380 & 51.73629 & $1.39 \mathrm{e}-07$ & -7.309854 & -6.310701 & -7.004403 \\
3 & 144.4564 & 27.15225 & $6.24 \mathrm{e}-08$ & -8.175455 & -6.748093 & -7.739096 \\
4 & 150.5848 & 6.566211 & $8.74 \mathrm{e}-08$ & -7.970346 & -6.114775 & -7.403079 \\
5 & 175.7975 & $21.61085^{*}$ & $3.48 \mathrm{e}-08^{*}$ & $-9.128393 *$ & $-6.844614 *$ & $-8.430214^{*}$ \\
\hline
\end{tabular}

注: *表明检验标准选择的最优滞后阶数。

在检验形式方面, 表 3 所表示的是以迹统计量和最大特征值为基础的 5 种模型形式的检验结 果。检验结果表明: 5 种模型形式都在 1\%的置信水平下通过 Johansen 协整检验, 但尚不能 确定最优模型。为确定最优模型形式, 对 5 种可能的协整形式均建立 VEC (Vector Error Correction Mode1) 模型, 然后根据 AIC 和 SC 最小信息准则确定 VEC 模型和协整检验的最优 形式。从表 3 中 AIC 和 SC 统计量的值可以看出, 两者均在第 4 种模型形式下值最小, 因此, 第 4 种协整检验形式即为 VEC 模型和协整检验的最优形式。与此相对应，反映变量之间长期 均衡关系的协整方程为 


$$
\mathrm{gc}_{\mathrm{t}}=\underset{[-5.500]}{0.108 \mathrm{fdi}_{\mathrm{t}}}+\underset{[-9.057]}{2.611 \mathrm{tr}_{\mathrm{t}}-\underset{[3.938]}{0.016 \mathrm{~T}}-8.834}
$$

式中 $\mathrm{T}$ 表示时间趋势, [] 内为 $\mathrm{t}$ 统计量, 以 $\Delta g c_{t}$ 为因变量的 VEC 估计结果为:

$$
\begin{aligned}
& \Delta g c_{t}=\underset{[3.816]}{0.211 E C M_{t-1}}+\underset{[0.302]}{0.066 \Delta c_{t-1}}+\underset{[0.879]}{0.246 \Delta c_{t-2}}-\underset{[-0.283]}{0.039 \Delta c_{t-3}}-\underset{[-2.831]}{0.350 \Delta g c_{t-4}}-\underset{[0.967]}{0.026 \Delta f i_{t-1}} \\
& -\underset{[-4.533]}{0.126 \Delta f d i_{t-2}}+\underset{[0.074]}{0.002 \Delta f d i_{t-3}}+\underset{[1.299]}{0.033 \Delta f d i_{t-4}}+\underset{[2.068]}{0.214 \Delta t r_{t-1}}+\underset{[4.819]}{0.309 \Delta t r_{t-2}} \\
& +\underset{[2.666]}{0.206 \Delta t r_{t-3}}+\underset{[3.494]}{0.188 \Delta t r_{t-4}}+\underset{[2.062]}{0.010}
\end{aligned}
$$

\begin{tabular}{|c|c|c|c|c|c|}
\hline 序列空间 & 无附加项 & 无附加项 & 有线性趋势 & 有线性趋势 & 二次趋势 \\
\hline \multirow{2}{*}{ 协整方程 } & 无截距 & 有截距 & 有截距 & 有截距 & 有截距 \\
\hline & 无线性趋势 & 无线性趋势 & 无线性趋势 & 有线性趋势 & 有线性趋势 \\
\hline 迹统计量 & 1 & 2 & 1 & 1 & 1 \\
\hline 最大特征根 & 1 & 2 & 1 & 1 & 1 \\
\hline AIC & -7.419 & -8.537 & -8.774 & -8.852 & -8.843 \\
\hline $\mathrm{SC}$ & -5.424 & -6.491 & -6.633 & -6.664 & -6.559 \\
\hline
\end{tabular}

此式子中，误差修正项 $\mathrm{ECM}_{\mathrm{t}-1}$ 的系数为证，这说明，其具有正向调节机制。

表3. Johansen协整关系检验结果汇总

注:（1）协整关系个数根据 McaKinnon-Haug-Michelis (1999) 的 1\%临界值确定;

(2) AIC 与 SC 是对相应应误差修正模型 VEC 整体进行诊断的最小信息准则统计量。

\section{Granger因果检验}

4.1 短期效应分析

\begin{tabular}{|c|c|c|c|c|c|c|}
\hline \multirow{2}{*}{ 检验对象 } & \multicolumn{2}{|c|}{$\triangle(\mathrm{fdi}) \mathrm{t}-1$} & \multicolumn{2}{|c|}{$\triangle(\mathrm{gc}) \mathrm{t}-1$} & \multicolumn{2}{|c|}{$\triangle(\mathrm{tr}) \mathrm{t}-1$} \\
\hline & $x^{2}$ & 效应 & $\chi^{2}$ & 效应 & $x^{2}$ & 效应 \\
\hline $\begin{array}{l}\triangle(\mathrm{fdi}) \mathrm{t}-\mathrm{i}, \\
(\mathrm{i}=1,2,3,4)\end{array}$ & & & $\begin{array}{l}26.221 \\
(0.000)\end{array}$ & - & $\begin{array}{l}48.621 \\
(0.000)\end{array}$ & - \\
\hline $\begin{array}{l}\triangle(\mathrm{gc}) \mathrm{t}-\mathrm{i}, \\
(\mathrm{i}=1,2,3,4)\end{array}$ & $\begin{array}{l}17.102 \\
(0.002)\end{array}$ & + & & & $\begin{array}{c}22.119 \\
(0.0002)\end{array}$ & - \\
\hline $\begin{array}{l}\Delta(\operatorname{tr}) \mathrm{t}-\mathrm{i}, \\
(\mathrm{i}=1,2,3,4)\end{array}$ & $\begin{array}{l}15.384 \\
(0.004)\end{array}$ & + & $\begin{array}{l}37.293 \\
(0.000)\end{array}$ & + & & \\
\hline
\end{tabular}

表 4 列出了基于 VEC 的短期 Granger 因果关系的检验结果。若各变量的 $\times 2$ 统计量在 5\%水平 上显著，将列出其相应短期效应的正负符号。

表4.基于误差修正模型的短期Granger因果关系检验结果

注: (1) ( ) 内为 $\times 2$ 统计量的伴随概率;

(2) 效应正记做 “+”、负记做 “-”。

第一, 政府最终消费、对外贸易是中国直接投资流入的 Granger 的原因。具体而言, 政府最 终消费与最终消费, 出口总额与进口总额的比重的上升都在短期之内显著的作用于直接投资 流入中国, 并且对直接投资的流入起到积极的作用。由于本文使用政府最终消费来整体测量 政府的活动, 反映政府的规模、职能力度、政策变化等。中国政府坚持改革开放的政策, 在 初期吸引外资方面给予外商投资者税收优惠、职工福利优惠、银行贷款等政策优惠, 通过法 律法规给予外商投资者投资环境保障，促使外商投资者在华投资，因此，政府最终消费对于 直接投资的流入的短期正效应符合预期; 本文使用出口总额与进口总额的比重来反映我国的 
对外贸易, 这种比重的上升即净出口相对规模的上升在内优化了要素配置效率, 提升了市场 运行效率, 加强了出口贸易企业的市场参与度, 在外加强了我国产品竞争力, 提升我国的企 业竞争力和形象, 这些都有助于吸引投资者的眼光。因此, 短期内出口导向作用越强, 越有 助于直接投资流入。

第二，直接投资流入、对外贸易是政府最终消费的短期 Granger 原因，它们在短期效应的符 号方向上正负不一：一方面，直接投资的流入的上升会降低政府最终消费在最终消费的比重; 另一方面, 净出口相对规模的上升在短期内提升了政府最终消费, 挤出了私人最终消费。从 原因上来说, 直接投资流入主要进入市场部门, 而不是政府部门, 因此其与政府最终消费短 期内负效应符合经济常态，也就是说，直接投资的流入在短期之内有助于政府职能的转换， 有助于政府的市场化程度的发展; 净出口能力的提高能够促进经济对外发展, 提升我国的世 界经济地位, 在内促进经济宏观增长, 调高最终消费水平, 因此, 对外贸易短期内与政府最 终消费具有正向关系也是极有可能的。

第三，直接投资流入、政府最终消费是对外贸易的短期 Granger 原因，都为短期负效应。从 短期来看, 直接投资流入会对我国的对外贸易产生障碍, 即短期内, FDI 与对外贸易具有替代 关系; 政府最终消费占最终消费的比重越高越不利于净出口相对规模的提升, 不利于对外贸 易的发展, 这点也符合经济规律, 就目前宏观发展看, 只有政府的市场化程度越高才会越利 于促进对外贸易的发展。

4.2 长期效应分析

1. 长期直接效应分析

表 5 给出了模型中每个变量作为因变量的协整方程的估计结果。除了 $\mathrm{fdi}$ 方程和 $\mathrm{tr}$ 方程中政 府最终消费 (gct) 外, 其他系数都在 $5 \%$ 水平下显著。在 gc 方程中, 直接投资流入（fdit） 和对外贸易 (trt) 都与政府最终消费 (gct) 显著的负相关。

表5. 基于协整方程的长期直接效应检验结果

\begin{tabular}{|c|c|c|c|}
\hline 因变量 & fdit & gct & trt \\
\hline fdit & & $\begin{array}{c}0.108 \\
{[-5.500]}\end{array}$ & $\begin{array}{c}-0.041 \\
{[9.077]}\end{array}$ \\
\hline gct & $\begin{array}{c}9.237 \\
{[-1.346]}\end{array}$ & & $\begin{array}{c}0.383 \\
{[-1.325]}\end{array}$ \\
\hline $\operatorname{trt}$ & $\begin{array}{c}-24.117 \\
{[15.183]}\end{array}$ & $\begin{array}{c}2.611 \\
{[-9.057]}\end{array}$ & \\
\hline $\mathrm{T}$ & $\begin{array}{c}-0.147 \\
{[-1.767]}\end{array}$ & $\begin{array}{c}0.016 \\
{[3.838]}\end{array}$ & $\begin{array}{c}-0.006 \\
{[-1.764]}\end{array}$ \\
\hline $\mathrm{C}$ & -81.601 & 8.834 & -3.384 \\
\hline Adj. R-squared & 0.572 & 0.799 & 0.905 \\
\hline
\end{tabular}

注: （1） T 表示趋势项, C 表示截距项;

(2) []中的内容表示相应系数的 $\mathrm{t}$ 统计量值。

通过对长期直接效应（表 5）与短期效应（表 4) 进行比较, 可发现: 在 gc 方程中, 对外贸 易（trt）的长期直接效应与短期效应正负符号保持一致，直接投资流入 (fdit) 的长期直接 效应与短期效应的正负符号发生了逆转。FDI 流入短期之内可以改善我国中小企业的对外发 展, 从而增加就业, 提高产品竞争力, 进而促进我国的整体经济发展; 但是 FDI 长期之内的 大量流动, 可能会造成 “飞鸟经济” , 这就会使得政府采取政府干预市场措施, 因此可能会 有政府与市场的界限难以理清的局面。

此比较结果也让我们看到: 短期的积极效应可能在长期之内转化为消极效应, 长期的正面效 应可能需要很多的短期负面效应来实现。这种辩证关系会使得中国经济升级过程短期与长期 发展出现政策难以抉择的局面。 


\section{3 长期总效应分析}

表 6 同时给出了两种意义的长期总效应的检验结果。第一种意义的长期总效应是检验长期均 衡的协整关系与因变量之间是否具有关系, 若其之间存在关系, 这种长期均衡的协整关系是 否显著影响因变量。可以根据误差修正项 ECMt-1 的系数在统计上是否显著的方法进行判断, 若显著则表明误差修正项或长期均衡的协整关系是因变量的长期 Granger 原因, 反之亦然。 表 6 的最后一行列出了 3 个误差修正模型的误差修正项 ECMt-1 系数的 $\mathrm{t}$ 统计量及其伴随 概率, 结果显示: 在 $5 \%$ 置信水平下, ECMt -1 系数在因变量为 $\triangle \mathrm{gct}$ 和 $\triangle \operatorname{trt}$ 的误差修正模型 中显著为正。这就可以说明长期均衡的协整关系是政府最终消费和对外贸易的长期 Granger 原因，但这种长期均衡的协整关系对直接投资流入却没有显著的长期效应。

第二种意义的长期总效应是检验长期均衡的协整关系与特定自变量共同是否对因变量产生影 响的综合反映, 检验方法是将 ECMt-1 与特定自变量的各期滞后项一起进行 Wald 联合显著检 验。表 6 中, 如果长期总效应在 1\%置信水平上通过 Wald 联合显著检验, 就在 “收敛值” 一 栏列出其长期总效应的符号和大小。

\section{表6. 基于误差修正模型的长期效应检结果}

\begin{tabular}{|c|c|c|c|c|c|c|}
\hline \multirow[b]{2}{*}{ 检验对象 } & \multicolumn{2}{|c|}{$\triangle($ fdi $) t-1$} & \multicolumn{2}{|c|}{$\triangle(\mathrm{gc}) \mathrm{t}-1$} & \multicolumn{2}{|c|}{$\triangle(\mathrm{tr}) \mathrm{t}-1$} \\
\hline & $\begin{array}{c}\mathrm{F} \text { 值 } \\
\text { (伴随概率) }\end{array}$ & $\begin{array}{c}\text { 收敛 } \\
\text { 值 }\end{array}$ & $\begin{array}{c}\mathrm{F} \text { 值 } \\
\text { (伴随概率) }\end{array}$ & $\begin{array}{c}\text { 收敛 } \\
\text { 值 }\end{array}$ & $\begin{array}{c}\mathrm{F} \text { 值 } \\
\text { (伴随概率) }\end{array}$ & 收敛值 \\
\hline $\begin{array}{c}\text { ECMt-1, } \Delta(\text { fdi }) \mathrm{t}- \\
\mathrm{i}, \\
(\mathrm{i}=1,2,3,4)\end{array}$ & & & $\begin{array}{l}9.25402 \\
(0.0005)\end{array}$ & 0.003 & $\begin{array}{l}32.809 \\
(0.000)\end{array}$ & +0.026 \\
\hline $\begin{array}{c}\text { ECMt- } 1, \quad \Delta(\mathrm{gc}) \mathrm{t}-\mathrm{i}, \\
\quad(\mathrm{i}=1,2,3,4)\end{array}$ & $\begin{array}{c}3.420 \\
(0.032)\end{array}$ & & & & $\begin{array}{c}30.34093 \\
(0.000)\end{array}$ & -0.017 \\
\hline $\begin{array}{c}\text { ECMt- } 1, \quad \triangle(\text { tr }) \mathrm{t}-\mathrm{i}, \\
(\mathrm{i}=1,2,3,4)\end{array}$ & $\begin{array}{c}3.121 \\
(0.042)\end{array}$ & & $\begin{array}{c}9.110 \\
(0.0005)\end{array}$ & -0.007 & & \\
\hline ECMt-1 & $\begin{array}{c}0.658 \\
(0.431)\end{array}$ & & $\begin{array}{l}14.560 \\
(0.002)\end{array}$ & & $\begin{array}{c}59.49401 \\
(0.000)\end{array}$ & \\
\hline
\end{tabular}

注:（1）（）内为Wald 联合显著检验 $\mathrm{F}$ 统计量的伴随概率;

(2) 收玫值是对特定自变量施加一个标准差的信息冲击, 广义脉冲响应函数因变量稳定之后 的取值;

(3) 所有广义脉冲响应函数均收敛于某一常数。

第一，直接投资流入与对外贸易都是政府最终消费的长期 Granger 原因，但直接投资流入的 长期总效应为正, 净出口相对能力的长期总效应为负。通过长期总效应与短期效应的对比发 现, 直接投资流入与净出口相对能力对政府最终消费的效应正负符号发生了逆转。直接投资 流入在长期发展中促进了政府的政府最终消费，挤出来私人最终消费，这是违背了经济的发 展趋势的; 净出口相对规模对政府职能、规模的长期影响上具有阻碍作用, 这是符合净出口 相对规模提高会促进市场企业、产品、要素等的发展, 从而增加了私人最终消费, 降低政府 最终消费的经济规律。

第二, 直接投资流入与政府最终消费都是对外贸易的长期 Granger 原因, 且与短期效应正负 符号保持一致。无论从短期还是长期来看, FDI 与对外贸易之间具有替代作用。

\section{5. 结论与分析}

利用 1980-2016 年中国的年度经济数据, 本研究发现在 FDI 流入、对外贸易与政府最终消费 之间存在长期均衡协整关系。根据短期与长期 Granger 因果关系的检验结果可看出以下关系。 第一, 从短期效应看, FDI 流入和对外贸易都是政府最终消费的 Granger 原因, 并且 FDI 与 贸易短期内具有替代关系, 对外贸易能够促进 FDI 流入。短期效应中，对外贸易的发展会促 
进 FDI 流入。但在长期直接效应效应中, 其对于 FDI 流入是具有阻碍作用。这就使得选择同 时使用出口与国外投资的政策内部协调难度变大。

第二, 短期效应与长期总效应之间存在矛盾。从短期效应看, 直接投资流入阻碍着政府最终 消费，对外贸易促进政府最终消费，但是在长期效应中，效应正负号均发生了逆转。这说明， 短期内 FDI 流入能够促使政府职能转换, 但是长期职能却阻碍政府职能的转换。短期与长期 之间存在的这种矛盾会使政府在选择使用政策的短期目标与长期目标处于两难之中。

第三，长期直接效应与长期总效应之间存在矛盾。以对外贸易为例，从长期直接效应看，对 外贸易促进政府最终消费, 与短期效应反映的相一致, 但是从长期总效应看, 对外贸易阻碍 政府最终消费。这种短期与长期矛盾使得政府与市场之间的关系更为复杂化。

根据经济规律, 在出口导向发展模式中, 直接投资流入会刺激我国的外贸企业的发展, 从而 刺激消费，因而在总消费中，私人消费比率将上升，但是在实证分析中发现，长期发展内， 直接投资流入却是抑制私人消费的比率上升，并不利于政府职能的转换。这可能是中国体制 二元化的原因。因此, 在中国经济升级向经济强国的过程中, 需要进行制度革新来处理好政 府与市场、政府与社会之间的关系, 通过转变政府职能, 创新政府管理, 发展市场竞争来完 善现代市场体系，使政府处于 “裁判员” 身份，在资源配置起到关键性作用，从而避免政府 直接干预经济。

\section{Acknowledgments}

Innovation Research on FDI inflow, foreign trade and government consumption expenditure based on Granger causality test model (SMXY201712)

\section{References}

[1]. Mundell, R.A. "International Trade and Factor Mobility." American Economic Review, 1957, (47):321-335.

[2]. Cameron, A.S. "The Size and Composition of Government Expenditure." Journal of Public Economics, 2007, (91):2230-2260.

[3]. Dreher, D. \& Sturm, J. \& Ursprung, W. "The Impact of Globalization on the Composition of Government Expenditures: Evidence from Panel Data.” Public Choice, 2008, (134):263-292.

[4]. Gemmell, N. \& Kneller, N. \& Sanz, L. "Foreign Investment, International Trade and Size and Structure of Public Expenditures.” European Journal of Political Economy, 2008, (24):151-171.

[5]. Huiai Yuan \& Lihong Zhao \& Chenhan Yuan. Research on the Structure of Human Capital and the Choice of Technical Level and the Motive Force of Economic Growth in Xi'an[A]. Research Institute of Management Science and Industrial Engineering.Proceedings of 2017 2nd International Conference on Education,Sports,Arts and Management Engineering(ICESAME 2017)[C].Research Institute of Management Science and Industrial Engineering:,2017:5.”

[6]. Huiai Yuan. FDI inflows,foreign trade, and government final consumption-based onemprical research after China's reform and opening up[D] JiLin University of Finance and Economics,2015. 